\title{
Magnetic Susceptibility of Compressed Rocks
}

\author{
Takesi NAGATA \\ Department of Earth and Planetary Sciences, University of Pittsburgh
}

(Read, Nov. 6, 1965 : Received, Nov. 15, 1965)

\begin{abstract}
The effect of uniaxial compression $(\sigma)$ upon the initial magnetic susceptibility $(\kappa(\sigma))$ of rocks is empirically expressed as $\kappa(\sigma)=\kappa_{0} /(1+\beta \sigma)$ where $\kappa_{0}$ denotes the susceptibility for $\sigma=0$. This empirical relation is interpreted as due to the effect of induced magnetoelastic energy upon rotation of magnetization of magnetic minerals whose initial anisotropy axes are randomly oriented. Feasibility of theoretical results are experimentally examined using titanomagnetite samples of different grain sizes.
\end{abstract}

\section{Introduction}

Dependency of magnetic susceptibility of rocks and assemblages of magnetic minerals upon uniaxial compression was experimentally studied by Kalashnikov and Kapitsa (1952) and by Grabovsky and Parkhomenko (1953) and Kapitsa (1955). The same phenomenon has recently been re-examined by Nagata and Kinoshita (1965) in connection with dependency of irreversible remanent magnetization of these materials upon uniaxial compression. In all cases of these experimental results, the relation of magnetic susceptibility $(\kappa)$ to uniaxial compression $(\sigma)$ is expressed by a hyperbolic form such as

$$
\kappa=\frac{\kappa_{0}}{1+\beta \sigma}
$$

where $\kappa_{0}$ denotes the magnetic susceptibility without compression and $\beta$ is a constant dependent on characteristics of materials. The magnitude of $\beta$ for various natural rocks, measured by Kapitsa, ranges between $0.8 \times 10^{-4}$ and $1.3 \times 10^{-4} \mathrm{~cm}^{2} / \mathrm{kg}$. For assemblages of magnetic grains, Grabovsky and Park homenko showed that $\beta$ amounts to about $1.5 \times 10^{-3} \mathrm{~cm}^{2} / \mathrm{kg}$. Nagata and Kinoshita have also obtained almost the same value of $\beta$, namely, $\beta=1.6 \times 10^{-3}$ $\mathrm{cm}^{2} / \mathrm{kg}$.

Kern (1961) and Stacey (1962) proposed theoretical interpretations of the observed dependency of $\kappa$ on uniaxial compression $\sigma$. In the Kern's theory, the specimen is considered to consist of a crystal aggregate with randomly oriented crystal axes, and consequently, neither magnetocrystalline anisotropy nor shape anisotropy of the specimen is apparently involved.

Starting on the Maxwell's relation among magnetoelastic strain tensor $\epsilon_{i j}$, stress tensor $\sigma_{i j}$, magnetizations vector $j_{k}$ and ambient magnetic field vector $H_{k}$, such as

$$
\left(\frac{\partial \epsilon_{i j}}{\partial H_{k}}\right)_{\sigma}=-\left(\frac{\partial j_{k}}{\partial \sigma_{i j}}\right)_{H}
$$


with the expression of magnetic susceptibility

$$
\chi_{m n}=\frac{\partial j_{m}}{\partial H_{n}},
$$

and magnetoelastic strain $(\epsilon)$ of isotropic material

$$
\epsilon=\epsilon_{i j} l_{i} l_{j}=\frac{3 \lambda_{s}}{2}\left\{\left(\alpha_{i} l_{i}\right)^{2}-\frac{1}{3}\right\},
$$

where $\alpha_{i}$ and $\lambda_{s}$ are direction cosine of magnetization and isotropic magnetostriction coefficient, Kern derived a general equation of $\chi$ as

$$
\left(\frac{\partial^{2} \chi}{\partial \dot{\sigma}^{2}}\right)_{H}+6\left(\frac{3 \lambda_{s}}{J_{s}^{2}}\right) \chi\left(\frac{\partial \chi}{\partial \sigma}\right)_{H}+4 \chi^{3}\left(\frac{3 \lambda_{s}}{J_{s}^{2}}\right)=0
$$

where $J_{s}$ denotes the spontaneous magnetization.

The general solution of ( 5 ) may be expressed as

$$
\chi=\frac{\chi_{0} C_{1}}{1+\beta \sigma}+\frac{\chi_{0} C_{2}}{1+2 \beta \sigma},
$$

where $C_{1}+C_{2}=1, \beta=3 \lambda_{s} \chi_{0} / J_{s}^{2}$ and $\chi_{0}$ denotes susceptibility for no compression.

Kern has further shown that $C_{2} \ll C_{1}$ for the initial susceptibility, and consequently, the dependency of initial susceptibility on $\sigma$ is given by

$$
\chi(\sigma)=\frac{\chi_{0}}{1+\beta \sigma}, \quad \beta \equiv \frac{3 \lambda_{s}}{J_{s}^{2}} \chi_{0} .
$$

This theoretical result is in agreement with the empirical expression given by ( 1 ).

Stacey approached the problem in a somwhat simplified and different way. In his theory, emphasis is given to the effect of magnetoelastic energy caused by $\sigma$ upon the rotation of magnetization which initially lies along the easy magnetization axes [111] when $\sigma=0$ and $H=0$. The rotational magnetic susceptibility $\left(\chi_{\perp}\right)$ for a special case that magnetic field $H$ is perpendicular to the easy direction can thus be easily obtained. Assuming that the susceptibility $\chi_{\|}$for the direction along the easy axis is equal to $\chi_{\perp}$, Stacey derived the following expression ;

$$
\frac{\chi(\sigma)}{\chi_{0}}=\frac{\frac{2}{3}\left|K_{1}+\frac{1}{3} K_{2}\right|+\frac{1}{2} N J_{s}^{2}}{\frac{2}{3}\left|K_{1}+\frac{1}{3} K_{2}\right|+\frac{1}{2} N J_{s}^{2}+\frac{3}{2}\left|\lambda_{s} \sigma\right|},
$$

where $K_{1}$ and $K_{2}$ denote magnetocrystalline anisotropy constants of cubic minerals and $N$ stands for the demagnetizing factor of magnetic minerals in rocks. If we put $\frac{3}{2} \lambda_{s} \sigma /\left\{\frac{2}{3} \mid K_{1}\right.$ $\left.+\frac{1}{3} K_{2} \mid+\frac{1}{2} N J_{s}^{2}\right\} \equiv \beta,(8)$ is identical to ( 7 ). Stacey particularly emphasized the importance of the effect of the demagnetizing field $N J_{s}^{2} / 2$ for rocks. These two theories look satisfactory to explain the observed dependency of $\kappa$ upon $\sigma$. However, when one goes further to consider the effect of uniaxial compression upon reversible susceptibility, together with that on irreversible magnetization, one may meet serious difficulty in interpreting even the 
$\kappa \sim \sigma$ relation, bzczuse no other anisotropy except the stress-induced anisotropy is explicitly considered in the Kern's theory and the assumption used in the Stacey's theory that $\chi_{\|}=\chi_{\perp}$ seems to be a little crude and artificial.

This paper will deal with the same problem in a different way which may also lead to a theoretical interpretation of the stress effect on remanent magnetization. Some simplifica tion is made for models adopted in this theory also. However, the physical meanings of the principal roles involved in this theory will be clear and intuitive.

\section{A Theory of the $\chi \sim \sigma$ relation}

The rotation of magnetization only is concerned in this theory. Since the initial magnetic susceptibility is concerned here, the applied magnetic field $(H)$ should be very small, and consequently, rotation of magnetization forced by $H$ from its initial direction of minimum energy is always small. As almost all experimental measurements were carried out with the condition that the direction of $\sigma$ is parallel to that of $H$, we may deal with such a case that $\sigma$ is parallel to $H$, and consequently, the physical phenomenon concerned is symmetric around the axis of $H$ direction.

Let angles between $\boldsymbol{H}$ and $\boldsymbol{J}_{s}$ and between $\boldsymbol{H}$ and the easy axis of some anisotropy $\left(K_{u}\right)$ be denoted by $\theta$ and $\varphi$ respectively. The anisotropy $\left(K_{u}\right)$ can be considered as due to magnetocrystalline anisotropy, or shape anisotropy, or even magnetoelastic anisotropy caused by randomly oriented internal stress. In any case, $\varphi-\theta=0$ at the initial demagnetized state when $\sigma=0$ and $H=0$.

In regard to individual magnetic domains, the total energy per unit volume in $H$ under $\sigma$ may be expressed as

$$
E(\varphi)=K_{u} \sin ^{2}(\theta-\varphi)+\frac{3}{2} \lambda_{s} \sigma \cos ^{2} \theta-J_{s} H \cos \theta+\text { constant }
$$

In our case, however, the direction of easy axis $(\varphi)$ with regard to that of $H$ is randomly distributed within a specimen. Then, the averaged value of the total energy with respect to all possible values of $\varphi$ will be given as

$$
\begin{aligned}
\bar{E} & =\frac{1}{4 \pi} \int_{0}^{2 \pi} d \lambda \int_{0}^{\pi} E(\varphi) \sin \varphi d \varphi \\
& =\frac{K_{u}}{3}\left(1+\cos ^{2} \theta\right)+\frac{3 \lambda_{s} \sigma}{2} \cos ^{2} \theta-J_{s} H \cos \theta
\end{aligned}
$$

The condition for minimum of $\bar{E}$ and the corresponding magnetization $(J)$ along $\boldsymbol{H}$ are then directly derived as follows:-
(i) If$$
\frac{J_{s} H}{\frac{2 K_{u}}{3}+3 \lambda_{s} \sigma}>1,
$$$$
\theta=0, \quad J=J_{s} ;
$$
(ii) if

$$
\frac{J_{s} H}{\frac{2 K_{u}}{3}+3 \lambda_{s} \sigma}<1,
$$




$$
\cos \theta=\frac{J_{s} H}{\frac{2 K_{u}}{3}+3 \lambda_{s} \sigma}, J=\frac{J_{s}^{2}}{\frac{2 K_{u}}{3}+3 \lambda_{s} \sigma} H .
$$

As we are interested only in magnetization derived by a amall value of $H$, the initial susceptibility $\boldsymbol{\kappa}(\sigma)$ is given directly from (13) as

$$
\kappa(\sigma)=\frac{d J}{d H}=\frac{J_{s}^{2}}{\frac{2 K_{u}}{3}+3 \lambda_{s} \sigma},
$$

and

$$
\kappa(\sigma=0)=\kappa_{0}=\frac{J_{s}^{2}}{2 K_{u} / 3}
$$

Putting

$$
\frac{9 \lambda_{s}}{2 K_{u}}=\frac{3 \lambda_{s} \kappa_{0}}{J_{s}^{2}} \equiv \beta
$$

(14) is rewritten as

$$
\frac{\kappa(\sigma)}{\kappa_{0}}=\frac{1}{1+\beta \sigma},
$$

which is in exact agreement with ( 7 ), and consequently, with the experimental formula ( 1 ).

In case of rocks, in which magnetic mineral grains are inevitably subjected to the demagnetizing field of $-N J$ expressed in terms of average demagnetizing factor $N$ of these grains, intensity of magnetization $J^{\prime}$ is expressed, instead of (13), as

$$
J^{\prime}=\frac{J_{s}^{2}}{\frac{2 K_{u}}{3}+3 \lambda_{s} \sigma+N J_{s}{ }^{2}} H
$$

and therefore, the initial susceptibility becomes

with

$$
\kappa^{\prime}(\sigma)=\frac{J_{s}^{2}}{\left(\frac{2 K_{u}}{3}+N J_{s}^{2}\right)+3 \lambda_{s} \sigma}=\frac{\kappa_{0}^{\prime}}{1+\beta^{\prime} \sigma},
$$

This is the case emphasized by Stacey in expression ( 8 ).

If we assume that $K_{u}$ is subject only to magnetocyrstalline anisotropy, then we may put $K_{u} \approx|K|_{1}$. The observed values of $K_{1}$ and $\lambda_{s}$ of magnetite at $20^{\circ} \mathrm{C}$ (Syono 1965) are 1.36 $\times 10^{5}$ c.g.s. and $39 \times 10^{-6}$ c.g.s. respectively. From these quantities we get $\beta \approx 1.3 \times 10^{-9}$ c.g.s. $=1.3 \times 10^{-3} \mathrm{~cm}^{2} / \mathrm{kg}$, which is in fairly good agreement with the observed values for magnetite. In the case of rocks, $N$ usually ranges between 3 and 4 , its average value being $\bar{N}=3.4$ (Nagata 1961). Since $J_{s} \approx 5 \times 10^{2}$ c.g.s. for magnetite, $\beta^{\prime}$ is evaluated as $\beta^{\prime} \approx 1.25 \times 10^{-10}$ c.g.s. $=$ $1.25 \times 10^{-4} \mathrm{~cm}^{2} / \mathrm{kg}$. This theoretical value of $\beta^{\prime}$ also is in rough agreement with the experimental values obtained by Kapitsa (1955).

If magnetic minerals are very fine grained, forming single domain particles, their shape anisotropy becomes the dominant factor to determine the $K_{u}$ value. The shape anistropy of 
a particle having $M$ and $N$ in demagnetizing factor along their minor and major diameters is expressed as

$$
K_{u}=\frac{1}{2}(M-N) J_{s}^{2} .
$$

The numerical value of $M-N$ is of the order of magnitude of unity. Hence, $K_{u} \approx 10^{5}$ c.g.s. for fine grained magnetite. If these grains are of the needle-like or plate-like shape, $K_{u}$ amounts to nearly $10^{6}$ c.g.s., the contribution of shape anisotropy to $K_{u}$ becoming larger than that of magnetocrystalline anisotropy. In such a case, $\beta$ takes a certain value smaller than $1.3 \times 10^{-3} \mathrm{~cm}^{2} / \mathrm{kg}$.

Although Stacey (1962) takes into account the effect of $K_{2}$ in addition to that of $K_{1}$ of the magnetocrystalline anisotropy, the experimentally observed value of $K_{2}$ is always appreciably smaller than that of $K_{1}$ for titanomagnetites (e.g. Syono $1965, K_{1}=-1.36 \times 10^{5}, K_{2}=$ $-0.44 \times 10^{5}$ for magnetite), we may safely ignore $K_{2}$ in the present approximation.

The present theory is mathematically much simpler than Kern's theory, and is much more self-consistent than Stacey's, because no additional assumption is introduced into the present theory which is based simply on the concept of rotation of magnetization only.

\section{Some Experimental tests of theoretical results.}

(15) in the preceding section indicates that

$$
\frac{\beta}{\kappa_{0}}=\frac{3 \lambda_{s}}{J_{s}^{2}} .
$$

If the material is always the same so that material constants $\lambda_{s}$ and $J_{s}$ are kept invariant, then the ratio $\beta / \kappa_{0}$ must be kept constant regardless of possible changes in $\beta$ and $\kappa_{0}$ respectively owing, for example, to the difference of grain size. Fig. 1 illustrates examples of observed results of $\kappa(\sigma) / \kappa_{0}$ of two assemblages of magnetite of different grain sizes; $(a)$ grain diameters of $60 \mu \sim 500 \mu$ and $(b)$ grain diameters of $1 \mu \sim 60 \mu . \chi_{0}$ of $(a)$ sample is 0.134 $\mathrm{emu} / \mathrm{gr}$ and $\chi_{0}$ of $(b)$ sample is $0.050 \mathrm{emu} / \mathrm{gr}$. The $\beta$ value is obtained from the $\chi \sim \sigma$ curves in Fig. 1 as $\beta_{a}=1.2 \times 10^{-4} \mathrm{~cm}^{2} / \mathrm{kg}$ and $\beta_{b}=5.0 \times 10^{-5} \mathrm{~cm}^{2} / \mathrm{kg}$. Hence, $\frac{\beta_{a}}{\chi_{0 a}}=0.9 \times 10^{-3}$ and $\frac{\beta_{b}}{\chi_{0 b}}$ $=1.0 \times 10^{-3}$ in the same unit. These values of $\beta / \chi_{0}$ are kept approximately constant regardless of appreciably large differences in $\beta$ and $\chi_{0}$ between the two samples.

Using artificially made samples of titanomagnetite of $0.4 \mathrm{Fe}_{2} \mathrm{TiO}_{4} 0.6 \mathrm{Fe}_{3} \mathrm{O}_{4}$ in chemical composition, similar test experiments were carried out. In this case, $\chi_{0 a}=0.068 \mathrm{emu} / \mathrm{gr}, \chi_{0 b}$ $=0.038 \mathrm{emu} / \mathrm{gr}, \beta_{a}=1.9 \times 10^{-4} \mathrm{~cm}^{2} / \mathrm{kg}$ and $\beta_{b}=1.3 \times 10^{-4} \mathrm{~cm}^{2} / \mathrm{kg}$. Hence, $\beta_{a} / \chi_{0 a}=2.7 \times 10^{-3}$ and $\beta_{a} / \chi_{0 b}=3.4 \times 10^{-3}$. In this case also, the theoretically expected constancy of $\beta / \chi_{0}$ seems to hold approximately. However, the magnitudes of $\lambda_{s}$ and $J_{s}$ are appreciably different between magnitite $\left(\mathrm{Fe}_{3} \mathrm{O}_{4}\right)$ and 0.4 ulvöspinel titanomagnetite $\left(0.4 \mathrm{Fe}_{2} \mathrm{Ti} \mathrm{O}_{4} \cdot 0.6 \mathrm{Fe}_{3} \mathrm{O}_{4}\right)$. According to Nagata-Yamaai (Nagata 1962), $J_{s}$ of $x=0.4$ titanomagnetite in expression of $x \mathrm{Fe}_{2} \mathrm{TiO}_{4}$ $(1-x) \mathrm{Fe}_{3} \mathrm{O}_{4}$ is approximately half of that of pure magnetite. On the other hand, Syono (1965) has shown that $\lambda_{s}$ increases almost linearly with $x$ at least up to $x=0.6$. An interpolation of Syono's experimental results gives $\lambda_{s}=110 \times 10^{-6} \mathrm{C}$.g.s. for $x=0.4$ titanomagnetite. 


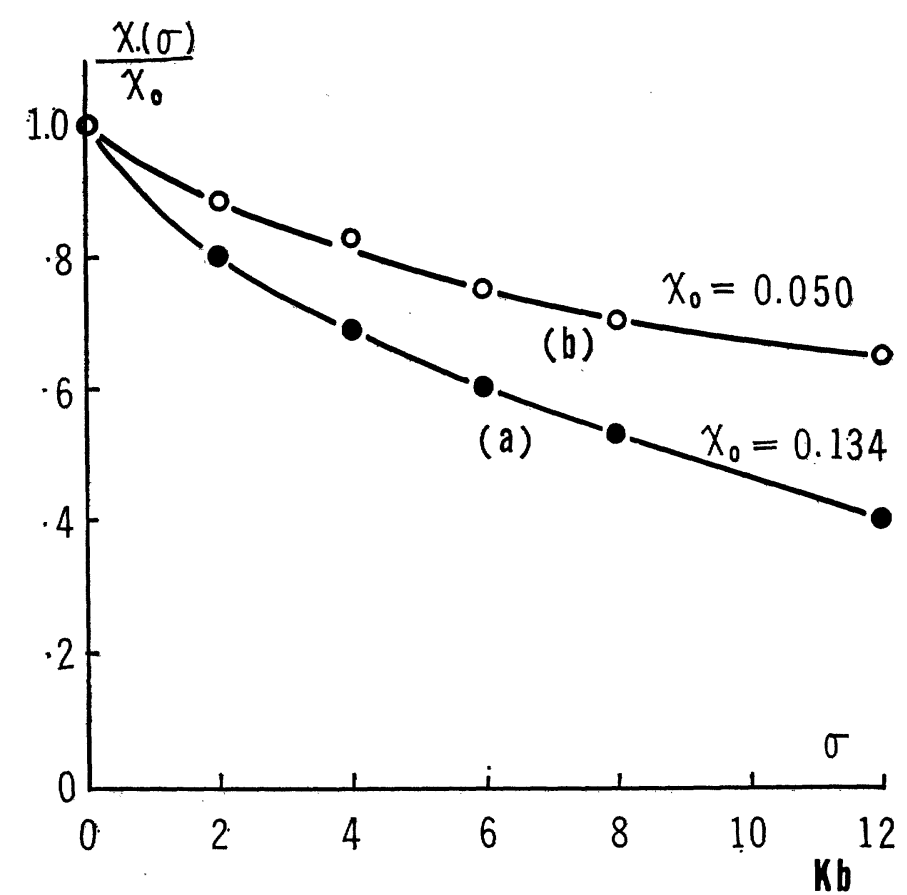

Fig. 1 Dependency of magnetic susceptibility of a magnetite grain assemblege under uniaxial compression upon grain size.

From (15) in the preceding section, one can get

$$
\frac{\beta(x=0.4)}{\beta(x=0)}=\frac{\kappa_{0}(x=0.4)}{\kappa_{0}(x=0)} \frac{\lambda_{s}(x=0.4)}{\lambda_{s}(x=0)} \frac{J_{s}^{2}(x=0)}{J_{s}^{2}(x=0.4)} .
$$

Putting numerical values of $\lambda_{s}$ and $J_{s}$ into (22), one may theoretically expect that

$$
\frac{\beta(x=0.4)}{\beta(x=0)}=11.3 \frac{\kappa_{0}(x=0.4)}{\kappa_{0}(x=0)} .
$$

For the large and small grain size samples, however, we have experimentally obtained $\beta_{a}(x=0.4) / \beta_{a}(x=0)=1.6, \beta_{b}(x=0.4) / \beta_{b}(x=0)=2.6$, and $\chi_{0 a}(x=0.4) / \chi_{0 a}(x=0)=0.51, \chi_{0 b}(x=$ $0.4) / \chi_{0 l}(x=0)=0.76$. Although the theoretically expected tendency that $\beta$ will increase with increasing $x$ in spite of decreasing $\kappa_{0}$ with increasing $x$ is qualitatively verified by the experiments, $(22)^{\prime}$ does not quantitatively hold at all. Therefore, one must notice a limit of applicability of the present theory. It seems almost certain that the disagreement between the theory and experiments is due to a simplified model of magnetization processes based only on the rotation of magnetization.

\section{Magnetic Susceptibility and irreversible magnetization of compressed rocks.}

It is assumed in the simplified model discussed in Section 2 that the averaged effect of anisotropy $\left(K_{u}\right)$ is represented by a uniform anisotropy energy obtained by averaging individual anisotropy energies of randomly oriented grains and domains. This assumption would be allowed as a rough approximation for dealing with initial magnetic susceptibility only. 
As Néel (1947) showed, however, the macroscopic magnetization of an assemblage of such magnetic grains should be an average of magnetizations of individual grains, in which the relative relationships among $J_{s}, \sigma$ and $H$ are different in different individual grains or domains. Then, irreversible magnetization is inevitably introduced in the average macroscopic magnetization.

Let us assume, for the sake of simplicity, a model of an assemblage of uniaxially anisotropic grains. Magnetizations parallel to the uniaxial easy axis and perpendicular to it being denoted by $J_{\|}$and $J_{\perp}$ respectively, the average magnetization $(J)$ will be approximately given by

$$
J \doteqdot \frac{1}{3}\left(J_{11}+2 J_{\perp}\right)
$$

Assuming that the direction of $\sigma$ is parallel to that of $H$, as in the case of Section 2, we may estimate the dependence of $J_{\|}$and $J_{\perp}$ on $\sigma$ as follows :-

$$
\frac{2 K_{u}}{3 \lambda_{s} \sigma}>1, \quad J_{\|}=J_{s} \text { for } H \geqq-\frac{2 K_{u}-3 \lambda_{s} \sigma}{J_{s}} ;
$$

if

$$
\left.\begin{array}{rl}
\frac{2 K_{u}}{3 \lambda_{s} \sigma}<1, J_{\|} & =\frac{J_{s}{ }^{2} H}{3 \lambda_{s} \sigma-2 K_{u}} \text { for }-\frac{3 \lambda_{s} \sigma-2 K_{u}}{J_{s}} \leqq H \leqq \frac{3 \lambda_{s} \sigma-2 K_{u}}{J_{s}}, \\
& =J_{s} \text { for } H \geqq \frac{3 \lambda_{s} \sigma-2 K_{u}}{J_{s}} ;
\end{array}\right\}
$$

and

$$
\left.\begin{array}{rl}
J_{\perp} & =\frac{J_{s}^{2} H}{3 \lambda_{s} \sigma+2 K_{u}} \text { for }-\frac{2 K_{u}+3 \lambda_{s} \sigma}{J_{s}}<H<\frac{2 K_{u}+3 \lambda_{s} \sigma}{J_{s}}, \\
& =J_{s} \text { for } H \geqq \frac{2 K_{u}+3 \lambda_{s} \sigma}{J_{s}} .
\end{array}\right\}
$$

Nagata and Kinoshita $(1964,1965)$ have experimentally shown that an increase of remanent magnetization by uniaxial compression in a magnetic field (piezo remanent magnetization) is mostly acquired by compression and then its release in presence of a magnetic field. The critical field $H_{c}$ for stable $J_{4}$ magnetization is given from (23) as

$$
H_{c}=\frac{2 K_{u}-3 \lambda_{s} \sigma}{J_{s}}
$$

When $\sigma=0$ and $H<2 K_{u} / J_{s}, J_{\|}$given by $J_{\|}=J_{s}$ or $-J_{s}$ for individual grains or domains is stable and does not contribute to the macroscopic magnetizations. Increase in $\sigma$, however, results in decrease of the critical field $H_{c}$, and those grains or domains for which $H_{c}<H$ are forced to make irreversible reversal of magnetization and then contribute to the macroscopic magnetization in such a way as given by (24). A decrease of $H$ down to zero in presence of $\sigma>2 K_{u} / 3 \lambda_{s}$ is accompanied by reversible magnetizations, as shown by (24), resulting in no remanent magnetization. However, a decrease of $\sigma$ down to zero in presence of $H$ results in an increase in $H_{c}$ up to $2 K_{u} / J_{s}$ and those grains or domains which were reversed by compression keep their $J_{\|}$magnetization as remanence. This remance of $J_{\|}$component is stable even when $H$ becomes zero. In actual specimens, those values of $K_{u}, \lambda_{s}$ and $J_{s}$ may not be unique but have some statistical distributions. 
The above-mentioned may be considered as an approximate qualitative interpretation of Nagata-Kimoshita's experimental results.

\section{Acknowledgment}

The writer's thanks are due to Mr. H. Kinoshita who actually carried out exprimental tescribed in Section 3 of this paper. This research work was supported by Grant NSF-G21592 and later by Grant GF-75 (US-Japan Scientific Cooperation Program) from the U.S. National Science Foundation.

\section{References}

Grabovsky, M.A. and E.I. Parkhomenko : Izvestia Ajad. Nauk. USSR. Geophys. Ser. 5, 405, 1953. Kalashnidov, A.G. and S.P. Kapitsa : Akad. Nauk. USSR, 86 (3), 521, 1952.

Kapitsa, S.P. : Izvestia Akad. Nauk. USSR., Geophys. Ser. 6, 489, 1955.

Kern, J.W. : J. Geophys. Res., 66, 3807, 1961.

Nagata, T. : "Rock Magnetism" rev. ed. pp 126-135, 1961, Tokyo.

Nagata, T. : Proc. Benedum Symp. Earth Magnetism, pp. 69-86., 1962, Pittsburgh.

Nagata, T. and H. Kinoshita : Nature, 204, 1183, 1964.

Nagata, T. and H. Kinoshita : J. Geomag. Geoele., 17, 121, 1965.

Néel, L. : C.R. Acad. Sci. Paris, 224, 1550, 1947.

Stacey, F.D. : Phil. Mag., 7, 551, 1962.

Syono, Y.: Japanese J. Geophys. 4, 71, 1965. 